\title{
PENSER L'ANTHROPOLOGIE DE LA LIBERTÉ AVEC SARTRE
}

Entretien avec Monique Selim, réalisé par Émir Mahieddin

\author{
Monique Selim, Emir Mahieddin
}

Association Française des Anthropologues | «Journal des anthropologues »

2021/1 n 164-165 | pages 151 à 163

ISSN 1156-0428

DOI 10.4000/jda.10758

Article disponible en ligne à l'adresse :

https://www.cairn.info/revue-journal-des-anthropologues-2021-1-page-151.htm

Distribution électronique Cairn.info pour Association Française des Anthropologues.

(c) Association Française des Anthropologues. Tous droits réservés pour tous pays.

La reproduction ou représentation de cet article, notamment par photocopie, n'est autorisée que dans les limites des conditions générales d'utilisation du site ou, le cas échéant, des conditions générales de la licence souscrite par votre établissement. Toute autre reproduction ou représentation, en tout ou partie, sous quelque forme et de quelque manière que ce soit, est interdite sauf accord préalable et écrit de l'éditeur, en dehors des cas prévus par la législation en vigueur en France. Il est précisé que son stockage dans une base de données est également interdit. 


\section{Journal des anthropologues}

Journal des

Association française des anthropologues

164-165 | 2021

Ethnographier la liberté

\section{Penser l'anthropologie de la liberté avec Sartre}

Entretien avec Monique Selim, réalisé par Émir Mahieddin

Revisiting the Anthropology of Freedom with Sartre

Monique Selim et Émir Mahieddin

\section{CpenEdition}

Journals

Édition électronique

URL : https://journals.openedition.org/jda/10758

DOI : 10.4000/jda. 10758

ISSN : 2114-2203

Éditeur

Association française des anthropologues

Édition imprimée

Date de publication : 3 mai 2021

Pagination : 151-163

ISSN : 1156-0428

Distribution électronique Cairn

\section{CAIRN INFO}

CHERCHER, REPÉRER, AVANCER

Référence électronique

Monique Selim et Émir Mahieddin, « Penser l'anthropologie de la liberté avec Sartre », Journal des anthropologues [En ligne], 164-165 | 2021, mis en ligne le 02 janvier 2024, consulté le 12 juillet 2021. URL : http://journals.openedition.org/jda/10758 ; DOI : https://doi.org/10.4000/jda.10758 


\section{PENSER L'ANTHROPOLOGIE DE LA LIBERTÉ AVEC SARTRE

\author{
Entretien avec Monique Selim' \\ Réalisé par Émir Mahieddin²
}

Émir Mahieddin - Quand nous avons initié le projet de ce numéro, tu as immédiatement évoqué l'œuvre de Jean-Paul Sartre, considéré comme le penseur par excellence de la liberté. C'est pour explorer les apports potentiels de la pensée sartrienne à cette anthropologie de la liberté que nous avons envisagé de réaliser cet entretien. Je propose de commencer par situer Sartre vis-à-vis du champ intellectuel français contemporain et de l'anthropologie.

En avril 2020, Anne Mathieu a publié un article dans Le Monde diplomatique dans lequel elle soulignait les attaques que subit Jean-Paul Sartre, au point que même le journal qu'il a fondé, Libération, ne lui a pas rendu hommage au quarantenaire de sa mort. Il y aurait un rejet, voire une détestation de Sartre assez répandue aujourd'hui. Cela dit, on voit par ailleurs qu'à la faveur des mouvements sociaux des années 2010, et notamment ceux de 2019 en Algérie, au Soudan, au Liban, au Chili, etc., les questionnements de Sartre sur les révolutions sont revenus sur le devant de la scène. Emmanuel Barot note ainsi un renouvellement de l'intérêt qu'il suscite à gauche dans les années récentes, après avoir été longtemps relégué aux oubliettes. C'est notamment son ouvrage Critique de la raison dialectique, qui proposait une synthèse de marxisme et d'existentialisme, qui fait l'objet d'une attention particulière et d'une redécouverte récente par la gauche. En tout cas, le refus de Sartre marque aussi l'anthropologie en particulier, jusqu'aujourd'hui.

Il faut souligner que Critique de la raison dialectique avait interpellé les anthropologues et les sociologues, dans la mesure où il traitait de problématiques

1 IRD-CESSMA, UMR 245 IRD, université Paris-Diderot, Inalco

Courriel : monique.selim@ird.fr

2 CéSor (Centre d'étude en sciences sociales du religieux)

Courriel : emirmahieddin@yahoo.fr 
qui les concernaient : comment les êtres humains se regroupent-ils et comment font-ils l'histoire tout en étant en retour forgés par ces groupements et agis par l'histoire ? Lévi-Strauss lui avait même consacré plusieurs séances de son séminaire au Collège de France (1960-1961) tout de suite après sa parution. Pour autant, et contrairement à d'autres philosophes français de premier plan tels que Michel Foucault, Gilles Deleuze ou Jacques Derrida, Jean-Paul Sartre a occupé une place marginale dans l'élaboration théorique de notre discipline. Il se fait assez rare. Il laisse une trace dans les études postcoloniales, du fait de ses engagements anticolonialistes et de ses essais sur le système colonial. Il faut mentionner notamment l'influence qu'il a eue sur l'œuvre de Frantz Fanon qui, à son tour, a inspiré Gayatri Spivak, Homi Bhabha, Achille Mbembe ou Edward Saïd, même si ce dernier reproche à Sartre sa position sur la Palestine. Ailleurs, il inspire une ethnographie phénoménologique au statut assez expérimental à quelques anthropologues scandinaves qui s'attachent à décrire des formes de présence au monde d'individus pris dans des situations d'ennui ou d'attente, des moments où « rien ne se passe ». Je pense à Billy Ehn et Orvar Löfgren ou plus récemment à Martin Demant Fredriksen. Enfin on le voit mobilisé, quoique trop rarement, dans des travaux d'anthropologie historique — je pense surtout aux travaux de Sophie Wahnich, très importants pour notre problématique. Mais Sartre reste finalement un auteur assez lointain pour la plupart des anthropologues.

Peux-tu revenir, en disant au passage quelques mots de ton propre rapport à l'œuvre de Sartre dans ton parcours intellectuel, sur la relation qu'entretient la discipline avec ce philosophe ? Quelles sont, à ton avis, les raisons de ce désamour ou de ce désintérêt des anthropologues pour Sartre?

Monique Selim - Sartre se situe hors de l'horizon de pensée de la très grande majorité des ethnologues dans les années 60-70 au cours desquelles s'affirme le rayonnement de l'existentialisme. Pour certains, tel Jean Benoist qui a fondé AMADES, l'association d'anthropologie médicale et de la santé, il restait l'objet d'un véritable honnissement, comme il me l'avait expliqué de vive voix alors que nous nous retrouvions à un colloque en Roumanie en 1999, période où l'influence de Sartre s'était déjà largement éclipsée. Hier comme aujourd'hui, Sartre est absent des références autant de ceux qui se disent anthropologues que de ceux qui chérissent les termes d'ethnographie et d'ethnologie. Tentons d'abord de comprendre cette coupure entre la sphère de l'anthropologie dans son histoire récente et la dernière manifestation d'un effort de construction d'un système philosophique au XXe siècle, si, comme moi, et malgré toute l'estime qu'il mérite, on ne considère pas Alain Badiou comme intégrant cette généalogie des fondateurs en philosophie. 
Il est important de noter d'abord que cette extériorité de la pensée sartrienne à l'anthropologie s'avère aussi forte dans le courant très largement dominant dans les années 60-70 du structuralisme que dans celui, minoritaire et contestataire, de l'anthropologie économique marxiste. Dans ces deux orientations, il y a en effet le point commun de réitérer une dichotomie entre les sociétés étudiées et les nôtres, assimilées à l'Occident. Une différence ontologique séparerait les deux types de société ; pour les structuralistes tout les oppose, l'historicité, le symbolique comme détermination primordiale, l'évolution dynamique et la fusion de l'individu dans la cohésion sociale; pour les marxistes, alors sous la coupe ou, au moins, sous la séduction du structuralisme althussérien, les modes de production font certes rentrer les sociétés étudiées dans l'histoire et le politique mais en oublient les acteurs dans leur dimension propre. La société d'appartenance de l'anthropologue est jugée à l'aune de son système politique démocratique, du libre arbitre et de la liberté des citoyens, attributs déniés à ces " autres " des tropiques et de la férule coloniale. Le politique, comme concept et comme engagement auprès des peuples colonisés et du tiers-monde, antagonise certes fortement ces deux courants à cette époque, où, il faut le rappeler, Jean-Marie Benoist, membre du club de l'horloge ${ }^{3}$ et du GRECE ${ }^{4}$, classés à l'extrême droite, est maître-assistant de Lévi-Strauss et où l'ethnologue Jacques Soustelle s'est porté avec l'OAS ${ }^{5}$ pour le maintien de l'Algérie française et a fait partie avec Salan et compagnie des amnistiés de De Gaulle.

J'en reviens maintenant, après ce détour indispensable, à Sartre qui, dans un petit ouvrage publié en 1946, affirme que "l'existentialisme est un humanisme ", englobant donc toute l'humanité et toutes ses composantes ici et ailleurs, dans la définition de ce qui fait la spécificité de l'homme, ce qui en soi est déjà une entreprise qui bouscule les anthropologues et leurs classements des sociétés et est de surcroît une qualification politique attribuée de façon universelle, ce qui vient heurter les anthropologues attachés de près ou de loin à la domination coloniale. Postuler que la conscience du néant, la fission dans l'être, le non-être, initient la liberté de l'homme, que celle-ci est toujours entière et entièrement partagée sous tous les cieux, rétablit le rapport entre ici et ailleurs et engage au sens fort du terme, engagement peu pratiqué dans l'anthropologie d'alors. Les quelques anthropologues qui ont lu et se sont nourris de Sartre sont de fait des anthropologues engagés qui soutiennent révoltes, révolutions et libération des tutelles coloniales et néocoloniales. Parmi ceux-ci, je citerai Gérard

3 Le Club de l'horloge, proche du Front national, est un cercle de réflexions qui, de 1974 à 2015, a prôné le national-libéralisme et l'identité de la nation.

4 Le Groupement de recherche et d'études pour la civilisation européenne est un think tank de la «nouvelle droite ", fondé en 1969.

5 L’Organisation de l'armée secrète qui a combattu l'indépendance de l'Algérie. 
Althabe qui reprend le concept sartrien de totalisation : l'individu est toujours séparé de lui-même, il est un projet d'être, aspirant à la transcendance et il est acteur de son histoire, agent de sa totalisation en lui. On en déduit immédiatement que l'aliénation est le produit de cette liberté intangible, inaliénable et Sartre nous invite finalement à comprendre en anthropologue les raisons que les sujets ont d'emprisonner leur liberté, de la forclore, de nouer Oppression et libération dans l'imaginaire selon le titre de l'ouvrage d'Althabe sur Madagascar. Sartre permet de restituer au plan théorique des subjectivités clivées dans les sociétés étudiées par les anthropologues, de comprendre que les rituels sont le fait d'individus portant au plus haut point la conscience des contradictions de leur condition, ce que les maîtres fous de Jean Rouch illustrent avec fulgurance.

Cette interprétation rapide de l'apport de Sartre à l'anthropologie que je développe avec toi est bien sûr le résultat de mon propre parcours et je conçois tout à fait que d'autres anthropologues de ma génération aient une toute autre lecture. Pour être brève et permettre le recul du lecteur, j'ai découvert Sartre très jeune, vers 12-13 ans, trouvant dans sa pensée une armature intellectuelle à ma vie, ce qui m'a conduit vers des études de philosophie avant de m'orienter vers l'anthropologie. Mes parents, très politisés (père énarque au Parti communiste, mère syndicaliste) m’ont abonnée à 15 ans, sur ma demande, au Temps modernes dans lequel j'ai puisé une partie de mes sources d'inspiration. J'avais 17 ans en 1968 et la figure de l'intellectuel qu'incarnait Sartre était pour moi un phare, celle du couple qu'il formait avec Simone de Beauvoir un modèle. À eux deux, ils donnaient toute légitimité au désir d'être libre et de s'exempter de toute norme sociale, politique, économique. Je me suis emparée de cette liberté.

É.M. - On peut le comprendre, car quelle liberté ! Pour Sartre, la liberté est ontologiquement inscrite au cœur de l'être. Certains philosophes, à l'instar de Philippe Cabestan, considèrent que la liberté sartrienne est d'une ampleur presque infinie. Cela tient d'abord au fait que sa définition de l'intentionnalité et de la conscience est différente de celle adoptée par la plupart des auteurs de sciences sociales. Pour Sartre, la conscience est un éclatement vers le dehors, un déploiement, plutôt qu'une intériorisation de ce qui nous entoure. On comprend qu'il ait mis les anthropologues mal à l'aise. Comme tu l'évoquais, Sartre s'est retrouvé confronté à un projet quasi-hégémonique dans la discipline dans les années 60-70, le programme structuraliste, qui consistait tout au contraire à mettre en lumière les structures inconscientes, les règles et les codes qui agissent les êtres humains à leur insu, voire les structures qui « les parlent " et "les pensent ". Les désaccords de fond avec les sociologues et les anthropologues de son temps ont culminé dans le débat très vif qui avait opposé Sartre à Lévi-Strauss qui l'attaque sévèrement dans La Pensée sauvage, un livre en partie conçu comme une réponse à Critique de la raison dialectique. Ce sont deux 
conceptions épistémologiques foncièrement différentes qui s'affrontaient là, engageant de nombreuses divergences qu'on ne peut malheureusement qu'évoquer rapidement ici : la distinction entre raison dialectique et raison analytique, la question de l'histoire et de l'historicité et la place de l'État, la place de l'action individuelle dans l'analyse du social, la relation de la conscience et de la pensée au réel, la possibilité d'agir du sujet dans le système, etc. La conscience a-t-elle le privilège d'agir sur le réel ou ce dernier ne constitue-t-il que la condition de possibilité de toute pensée et de la conscience elle-même ? Pierre Bourdieu s'en prendra aussi à Sartre à plusieurs reprises en dénonçant son "ultra-subjectivisme " ignorant le poids des conditions économiques et sociales. Il accusera la Critique d'être " un roman imaginaire de la mort et de la résurrection de la liberté ». Avec de tels adversaires institutionnels, on conçoit qu'oser se référer à Sartre en anthropologie pouvait relever d'une véritable gageure.

Symétriquement, Sartre reprochait au structuralisme son anti-historicisme et sa négation du sujet, qu'il voyait comme l'idéologie d'une civilisation technocratique. Il critiquait l'idée que penser la structure était suffisant, oubliant la praxis de l'agent qui fait l'histoire, à savoir l'humain libre, conscient et réflexif, et son serment au groupe. Nous aurions bien besoin d'une pensée d'inspiration sartrienne dans la séquence politique que nous traversons. Les structuralistes cherchaient en effet ce qui agit l'être humain depuis son dehors plus que la liberté en lui. Mais il faut insister sur ce que tu disais à l'instant sur la dialectique entre aliénation et liberté et sur les subjectivités clivées : Sartre ne niait aucunement le conditionnement de l'être humain par ce qu'il produit luimême, ce qu'il appelle le "pratico-inerte ». Il affirmait une « liberté décentrée ", certes ébranlée mais qui n'en est pas moins une liberté que les humains peuvent s'efforcer de reconquérir collectivement.

Au-delà des contrastes, il faut souligner, comme le fait Sophie Wahnich à raison, que Sartre comme les anthropologues se rejoignaient en ce qu'ils cherchaient à faire valoir l'égale dignité de toutes les voix. Mais en tout cas, dans toutes ces critiques et dans l'opposition à l'anthropologie sartrienne, on perçoit combien la conception de la liberté occupe une place fondamentale.

Cela pose immédiatement une question : une pensée qui accorde tant de place à la liberté est-elle compatible avec l'anthropologie ? James Laidlaw, en reprenant Zygmunt Bauman, dit par exemple de l'anthropologie qu'elle est une science de la "non-liberté ", attachée qu'elle est à débusquer les déterminismes sociaux, culturels et historiques. Peut-on être une anthropologue sartrien'ne?

M.S. - Considérer l'anthropologie comme une science de la non-liberté c'est, de mon point de vue, faire œuvre de réification de l'autre où qu'il se situe désormais dans un monde globalisé, numérisé, structuré par le marché capitaliste en 
perpétuel renouvellement. En réifiant l'autre on s'abstrait d'une situation partagée aujourd'hui sur laquelle l'autre est précisément plus que jamais un miroir. Dénier la liberté à l'autre, ne chercher que des déterminismes auxquels on échapperait en devenant anthropologue est une entreprise réactionnaire au sens propre du terme. Je reviens sur les rituels, objets ancrés s'il en est dans l'histoire de notre discipline anthropologique et qui ont été appréhendés par toute une partie de l'anthropologie symboliste comme hors histoire, immuables, ou alors donnant à voir des manifestations dégradées de leur forme originelle structurelle. En travaillant sur des rituels au Laos et au Vietnam, pays gouvernés par des États-parti communistes, j'espère avoir montré l'extraordinaire liberté que déploient les médiums et dans laquelle ils entraînent leurs adeptes. Au Laos, dans les années 90, sous le couvert de leurs génies réinventés, les médiums mettaient en scène dans leurs cérémonies improvisées un combat contre le gouvernement dictatorial qui semait la terreur ; ce combat était triomphalement gagné par les génies, incarnant une volonté de retour du marché, traduite aussi dans un marché des génies en pleine croissance. Liberté personnelle, liberté politique dans l'imaginaire, liberté économique dans les dépenses monétaires qu'entraînent les cultes, les rituels me semblent paradigmatiques de cette liberté qui définit la condition humaine chez Sartre. J'ajouterai que les entretiens que j'ai menés avec un immense plaisir avec les médiums, se mettant face à moi dans une extraordinaire liberté de relation et de parole, restent des leçons vivantes de ce que dit Sartre de la liberté comme absolue, apodictique.

Que l'anthropologie doive postuler et mettre en avant la liberté des sujets, et ce y compris sous les pires despotismes, me paraît nécessaire à l'analyse des dynamiques sociales et surtout politiques. Le politique est une production de la liberté, d'une hypothèse de liberté a priori, et une anthropologie qui occulte le politique comme ce fut le cas très souvent, en particulier avec la domination coloniale et néocoloniale en Afrique, est heureusement, me semble-t-il, aujourd'hui obsolète.

Je voudrais actualiser cette phrase de Sartre disant que "nous n'avons jamais été aussi libres que sous l'occupation " en la diffractant dans la période présente. En pleine crise sanitaire mondiale liée au coronavirus, alors que des mesures de confinement s'imposent un peu partout, aux USA, en Allemagne, en Italie et ailleurs, des gens défilent au nom de leur liberté contre le confinement, revendiquant la liberté d'être infecté et en Russie de telles manifestations se font virtuelles, en ligne. En tant qu'anthropologues, il nous faut être attentifs à ces manifestations même si elles sont des soutiens de Trump et de Poutine, et le fait que soit brandie l'idée de liberté dans ces circonstances me semble au cœur des interrogations que soulèvent votre appel à contribution pour ce numéro du Journal des anthropologues. La liberté, dans le cadre de la libéralisation de 
l'économie globale, retentit sur tous les champs sociaux et fait écho aux nouvelles subjectivités sur un mode d'inversion expiatoire. Quand Sartre prononce sa célèbre remarque, il s'agissait moins d'une condamnation des collaborateurs que de l'appel à déchiffrer comment des individus prenaient, dans une situation précise d'oppression, l'occasion de saisir pleinement leur liberté, contre toutes les apparences d'une scène déjà bloquée. Avec les manifestants américains et russes ce sera important, lorsque le temps des enquêtes reviendra, de replacer ce moment dans leurs trajectoires, de voir comment ils ont dévié librement, comment leur liberté s'est enlisée dans un mirage, comment ils ont sombré dans la déréliction. En d'autres termes, qu'est-ce qui les a poussés à une telle identification avec le pouvoir en place, à remettre en scène le vieux scénario de la guerre froide, le confinement étant dans leurs bouches une mesure " communiste " ? À un autre niveau, cette persistance d'une image du communisme comme antithétique de la liberté questionne d'autant plus actuellement que les États-parti communistes sont tous convertis au capitalisme, la Chine en tête comme leader de la globalisation du marché. La liberté d'expression fut, du temps de l'URSS, le drapeau politique du " monde libre " mais, aujourd'hui, la problématique est entièrement économique, une concurrence pour l'hégémonie, ce qui laisse penser que les manifestants américains se sont arrêtés au siècle dernier, un arrêt sur image quasi-psychotique.

É.M. - Si l'on adoptait une perspective sartrienne sur la liberté des sujets auprès desquels nous menons nos enquêtes de terrain, quelles seraient les implications pour l'anthropologie?

M.S. - Les implications d'une perspective de liberté des sujets sur l'anthropologie me paraissent avant tout méthodologiques et épistémologiques. Le terme d'informateur, qui a retrouvé une aura insensée aujourd'hui, me paraît le signe le plus emblématique d'une incapacité à penser la liberté des sujets dans une investigation anthropologique. Ce terme d'informateur est marqué par les configurations coloniales dans lesquelles l'anthropologue, légitimé par la situation de domination politique en vigueur, arrachait des informations à des individus sélectionnés pour leurs capacités intellectuelles, en position d'inégalité structurelle. Michel Leiris se rappelle avoir giflé un informateur qui ne répondait pas de façon satisfaisante à ses questions sur la parenté et l'alliance. L'anthropologue, désormais, pour mener à bien ses recherches, doit penser l'égalité des sujets avec lesquels il entre en dialogue pour comprendre les logiques qu'ils mettent en œuvre et plus généralement les axes des rapports sociaux en jeu dans leur société. Cette égalité est un principe symbolique et un ressort imaginaire de toute enquête réussie, car elle présuppose une égale liberté de la part des protagonistes à s'engager dans une relation qui va les lier dans une finalité de 
connaissance. La liberté en jeu de part et d'autre est la condition sine qua non pour produire une intelligibilité des situations et sa conséquence est immédiatement épistémologique : la connaissance n'est pas une addition d'informations mais un décryptage réflexif, en mouvement, qui creuse les tensions intrinsèques à une situation, les contradictions, les adhésions et les lignes de fuite qui la perforent. Pour revenir à la période présente, les pôles " mourir du virus " ou "mourir de faim » désignent bien les contraintes auxquelles les gens s'affrontent dans plusieurs pays, mais ces dernières n'expliquent en rien les cheminements des sujets, leurs choix, leurs hésitations, leurs balancements, qui relèvent de l'exercice de leur liberté.

\section{É.M. - Comment cela se traduirait-il dans la collecte et la restitution de données ? Comment peut-on faire transparaître le sujet libre dans l'écriture sur l'autre?}

M.S. - Dans le cours d'une investigation anthropologique, alors que des relations fortes se sont nouées, interviennent des moments récurrents où les sujets exposent à l'anthropologue le champ des possibles qui s'ouvre à eux, le prennent à témoin du carrefour où ils se trouvent, le sollicitent pour mieux penser leur liberté en acte. Face à de telles séquences dans lesquelles se cristallisent les imaginaires en jeu, la position de l'anthropologue est plus oxymorique que de coutume. De mon point de vue, vigilance, maitrise et rétention de son influence et de son jugement s'imposent alors pour renvoyer au sujet, en miroir, ses capacités de réflexivité propre sur sa vie et la conduite à tenir. Un bref exemple me permettra d'illustrer ma conception axiologique de ces moments de libération de la parole et de la pensée qui sont essentiels pour l'analyse des rapports sociaux dans le groupe étudié. Au Bangladesh, dans les années 89-90, je me suis immergée durant un an dans la filiale de la multinationale américaine Pfizer, jouissant grâce à son directeur bangladeshi de conditions exceptionnelles d'enquête auprès de toutes les couches d'employés de l'usine. La construction hiérarchique interne était le produit politique inédit de la guerre de libération de 1971 : les cadres qui avaient collaboré avec les Pakistanais pour le maintien de la domination coloniale du Bengale, étaient marginalisés. L'imam de la mosquée à l'intérieur du parc de l'usine était sous contrôle syndical et choisi pour son confinement dans une sphère religieuse détachée du politique, en continuité avec la première période de l'indépendance où les partis politico-religieux, favorables au Pakistan, avaient été interdits. Les ouvriers qui avaient lutté main dans la main avec leur directeur bengali avaient des salaires supérieurs aux cadres et leurs leaders syndicaux avaient été eux-mêmes, malgré leur très faible niveau d'éducation, élevés au rang de cadre, sauf un. Ce dernier, dont le pouvoir dans l'usine était très important et redoublé par ses hommes de main armés 
(ce qui est courant au Bangladesh) avait refusé cette promotion et s'était accroché à son poste d'ouvrier et syndical. Un jour, cet homme d'une cinquantaine d'années, imposant et redouté de tous, me demande de le suivre dans la salle de réunion de la direction où il a l'habitude de rentrer à sa guise, me fait asseoir et m'expose les raisons de ses choix antérieurs et le dilemme dans lequel il se trouve : doit-il ou non accepter enfin le poste de cadre qui lui est toujours offert ou continuer de refuser cette promotion ? Interloquée, je l'invite à parler plus mais refuse la perche du conseil qu'il me tend avec insistance. D'autres anthropologues auraient peut-être mis cet instant de confidence hors enquête et auraient accédé à sa demande, voyant en celle-ci un resserrement relationnel. Au contraire, j'ai voulu laisser entière la liberté de mon interlocuteur dans cette sorte de vide, de suspension angoissée où il se tenait à nu devant moi et pensait, qu'après avoir tellement écouté les uns et les autres, je me serais fait une opinion avisée de l'atmosphère de l'usine et que je le lui restituerai.

Venons-en à la deuxième partie de ta question : l'écriture est fondamentale mais l'anthropologie ne peut pas se cantonner dans les leurres épistémiques d'une supposée description - ce qui est une fiction — et céder aux narratives à l'américaine comme si la parole des sujets avait à elle seule valeur de vérité. Les discours des acteurs sont certes essentiels à restituer dès lors qu'ils sont analysés sur le fonds de l'observation des situations dans lesquelles ils sont émis, et en premier lieu la relation avec l'anthropologue qui doit être scrutée dans toute sa complexité de part et d'autre, pour éviter identifications et projections. La liberté de l'anthropologue me paraît néanmoins aussi totale que celle de ses interlocuteurs dès lors que ceux-ci sont prévenus avec le plus de transparence possible de l'entreprise de connaissance qui motive sa venue et ses questionnements. Pour ma part, à l'encontre des culpabilisations complaisantes et des sentiments de trahison que mettent en scène avec émotion des anthropologues dans leurs réticences à interpréter les discours recueillis, je considère légitime l'effort anthropologique de donner des clefs herméneutiques qui vont éventuellement contredire les sujets, aller à l'encontre de leur propre vision du monde. Quant à la restitution de la connaissance, elle est aujourd'hui incontournable, ne serait-ce que parce que l'anthropologue se meut autant que les acteurs dans un univers entièrement numérisé où la communication est globale et les réseaux sociaux omniprésents.

Cette quasi-obligation à la restitution n'est pas de l'ordre de la morale et ne peut être un frein à la tentative d'élucidation anthropologique. J'en donnerai juste un exemple pris en Ouzbékistan où, avec Laurent Bazin et Bernard Hours, nous avons mené en 2004 et 2005 des enquêtes dont nous avons choisi d'un commun accord de publier les résultats sans restriction aucune, à l'encontre d'une partie des chercheurs français travaillant dans ce pays sous dictature 
vivant dans une véritable obsession pour l'identité nationale, redoutant de ne plus se voir octroyer de visas à l'avenir. Nous avions été encouragés par les chercheurs de l'académie des sciences sur lesquels je m'étais concentrée et qui insistaient pour que je témoigne de l'oppression idéologique à laquelle ils étaient astreints et de leur condition misérable. Bien sûr, nous n'avons pas pu retourner en Ouzbékistan, ni même parler de notre livre publiquement à Paris, les différents lieux où devait avoir lieu la présentation ayant reçu des menaces de l'ambassade d'Ouzbékistan. Mais je pense que nous avons agi à la hauteur de l'espérance de liberté que nos interlocuteurs avaient placée en nous et, à mes yeux, c'est le principal. La servitude des Ouzbékistanais n'était pas à nos yeux une rente de situation de recherche.

É.M. - Cela nous ramène à la question de l'engagement. Selon toi, dans quelle mesure le modèle sartrien de l'intellectuel engagé, très critiqué aujourd'hui, peut-il inspirer un modèle contemporain d'engagement des anthropologues dans la cité ?

M.S. - La figure de l'intellectuel sartrien, soutenant toutes les luttes des opprimés ici et ailleurs est aujourd'hui caricaturée à l'extrême et refoulée au pôle négatif de l'intellectualité. Plutôt que d'idéaliser Sartre, il faut comprendre d'abord vers quel modèle on nous pousse en s'acharnant à nourrir des décennies plus tard une telle abomination à son égard : conseiller du prince, expert, fantoche médiatique, savant soi-disant neutre, synthétiseur normatif, moraliste, etc. Toutes ces catégories s'entremêlent aujourd'hui dans un marché de la reconnaissance qui paraît dérisoire si l'on se souvient que Sartre refusa le Prix Nobel. À un autre niveau, en s'émancipant des régimes de la contestation, l'engagement connaît depuis les années 2000 une mutation importante qui en fait une injonction morale et une plus-value économique. En effet, individuel et collectif, l'engagement est prescrit de tous côtés, dans le monde du travail et de l'entreprise comme, doit-on ajouter, dans les sciences sociales. Si les salariés doivent s'engager pour la réussite de l'entreprise, de façon plus subtile, on observe maintenant que l'engagement pour des causes nobles fait sortir des cadres d'entreprises classiques pour fonder leur entreprise engagée ou s'engager dans des entreprises engagées. Cet engagement général, subjectif et objectif, entrepreneurial, est salué par tous dans la perspective capitaliste puisqu'il constitue un tremplin professionnel comme le soulignent des analystes financiers. Moteur économique du marché à travers le formatage et la captation du désir, l'engagement est aussi un ressort essentiel du marché de la démocratie participative qui a désormais ses entrepreneurs, ses clients, ses plateformes et est réglé sur une concurrence qui s’accroît. 
Dans les sciences sociales, l'engagement est aussi une forme de retraduction de ce nouveau devoir à s'engager à tous moments pour faire progresser la société et il se présente aussi comme un engagement économique et une participation au marché à travers les nombreux dispositifs créés par de jeunes chercheurs auxquels les institutions publiques n'offrent plus de recrutement. L'engagement politique est certes présent mais vient en accompagnement — après ou de côté — de cette nécessité à s'engager pour construire des modes de subsistance et d'emploi originaux.

Il y a de nombreuses manières de s'engager en tant quanthropologue et certaines à mon avis, par l'identification au sujet-objet sur lequel elles se fixent, sont une négation de la recherche anthropologique en créant de la confusion et des projections antithétiques d'un travail d'analyse sociale. Le risque est d'autant plus grand pour les anthropologues que la connaissance est produite à partir des relations interpersonnelles développées et un passage sur le divan d'un analyste est toujours fructueux dans cette configuration très singulière. La segmentation des espaces, conduisant à un engagement du chercheur extérieur à son champ professionnel et à son activité d'investigation, me paraît notablement un leurre. L'engagement est néanmoins consubstantiel à une perspective d'anthropologie au présent car une science désengagée, soit décontextualisée et hors politique est un fantasme. Si l'objectivité est une illusion, l'objectivation relève d'un exercice de liberté qui nourrit l'horizon de l'anthropologue impliqué dans une époque et une situation donnée. Ainsi, il est induit à tenter de se dégager au maximum des normes idéologiques, méthodologiques et conceptuelles qui dominent dans sa propre société mais aussi à repérer les modes de conformité et de soumission dans le groupe sur lequel il se focalise, ce qui n’appelle pas le rôle héroïque de les en libérer.

\section{É.M. - Pour prendre un autre exemple d'anthropologie engagée, que donne une perspective sartrienne du point de vue d'une anthropologie travaillant à la libération des femmes?}

M.S. - Permets-moi d'abord d'être un peu réticente sur l'expression d'une « anthropologie travaillant à la libération des femmes ». Bien que féministe, je me méfie des bonnes intentions de libérer les uns ou les autres et il suffit de faire son travail d'anthropologue le plus honnêtement possible pour que ses résultats puissent être entendus par ceux et celles qui voudraient les utiliser en armes de lutte. Quand il s'agit des femmes, la prudence épistémologique est encore plus nécessaire maintenant où la cause des femmes est une affaire rentable, alimentant les politiques publiques et où l'État se veut le grand protecteur des femmes. Le genre est devenu le support de normes globales qui accompagnent dans ses mutations le capitalisme et qui entendent le moraliser sans toucher à ses 
structures économiques. Dans ces processus de moralisation, les femmes sont placées en tête, présentées comme des victimes face à des hommes assimilés à des prédateurs, la libération sexuelle des années 70 passant pour n'avoir profité qu'à ces derniers sans compter la criminalité sexuelle qu'elle aurait autorisée. La période contemporaine - marquée par un procès de 1968 qui n'en finit plus d'être scandé et qui frappe concomitamment le couple Sartre-Beauvoir, comme grands pervers - est donc paradoxale dans le champ des sexualités, libéralisant désirs et pratiques, incorporant l'image et en même temps opérant une dissolution et une resubstantialisation de la dualité sexuelle. La revalorisation des femmes par leurs capacités intrinsèques de soin généralisé en faveur de tous et de l'environnement, le care, va de pair avec une renaturalisation de leur être et, dans cette optique, la régression idéologique pointe sous des habits renouvelés. L'antinaturalisme fondamental qui sous-tend la conception de la liberté chez Sartre est actuellement fortement affaibli par la conscience partagée d'une destruction menaçante de l'environnement qui fait le lit de la redécouverte de la nature, terme qu'on croyait avoir déjà mis dans les poubelles de l'histoire au $\mathrm{XX}^{\mathrm{e}}$ siècle. La proximité supposée des femmes avec la nature, vieux précepte de justification de leur minoration, revient en force, auréolée des fonctions de gardiennes naturelles de l'environnement. La liberté et les droits des femmes n'ont jamais été aussi grands mais, dans le même moment, la requalification dont les femmes sont l'objet les renvoie à leurs fonctions premières, maternité, allaitement, service, famille et s'arracher à ces devoirs gratifiants pour reprendre sa liberté peut être bien difficile psychiquement. Tout se passe comme si les dépendances passées des femmes, repeintes aux vertus du moment, devaient servir à leur émancipation présente dans un jeu de dupes à mes yeux très périlleux. Mais bon nombre de mes jeunes collègues et amies s'inscrivent dans ce mouvement et je suis attentive à l'évolution de leurs analyses qui est un matériau de réflexion anthropologique. Les femmes sont notablement au cœur des nouvelles rhétoriques de la liberté sur lesquelles votre appel à contribution du Journal des anthropologues attire avec beaucoup de pertinence l'attention.

Je voudrais souligner pour conclure qu'il y a aujourd'hui une sorte de retournement de la liberté sur lequel il nous faut être vigilants en tant qu'anthropologues, mais aussi simplement en tant que sujets libres, quelle que soit notre condition sexuée. 


\section{RÉSUMÉ}

Cet entretien revient sur les relations de l'anthropologie à la pensée sartrienne et questionne son potentiel heuristique pour que la discipline s'empare de la question de la liberté.

Mots-clefs : Liberté, libération, sujet, Sartre, anthropologie et philosophie.

\section{SUMMARY}

\section{Revisiting the Anthropology of Freedom with Sartre}

This interview revisits the relationship between anthropology and Sartrean thought and questions its heuristic potential for the discipline's apprehension of the issue of freedom.

Keywords: Freedom, liberation, subject, Sartre, anthropology and philosophy. 